Principles of Posterior Fossa Surgery. 2012. Edited by Anil Nanda. Published by Thieme Medical Publishers, Inc. 252 pages. C\$140 approx.

\section{Rated tristror}

Anil Nanda, Chairman of the Department of Neurosurgery at Louisiana State University Health Sciences Center, assembled and edited this volume dedicated to the posterior fossa, and coauthored seven of its 25 chapters. As one of Peter Jannetta's former students and present day disciple she comes by his expertise in, and passion for, this anatomic territory honestly. This text is an earnest contribution to the

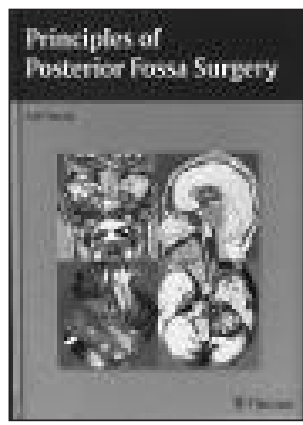
neurosurgical library, readable, thorough but not too thick. It begins with a concise review of the history of posterior fossa surgery followed by a richly illustrated chapter on microsurgical anatomy, nicely done. The remaining chapters in this section of the book, on imaging, pathology and nonsurgical conditions, are relatively brief and the latter two weakened by being entirely without illustrations (especially pathology). The next two multi-authored sections, entitled "Disease-Based Management" and "Surgical Techniques" contain unavoidable repetitions, not always a bad thing. Many chapters are concise, authoritative and excellent, but as expected, others less so. In total, tumours are well covered, as is infectious disease. Three common conditions on which the book disappointed were microvascular cross compression syndromes, dural arteriovenous fistulae of the posterior fossa and Chiari 1 malformations. Microsurgical approaches to aneurysms in the posterior circulation are well and amply covered by experts in the field, but the next edition of this book might find this topic included in the history chapter.

This book provides good, accessible information and principles for trainees, and is a reasonable price for a high quality publication. For more experienced surgeons the content is, on average, an adequate review. Of course the individual topics and subjects are covered elsewhere in more comprehensive neurosurgical texts, but this book is unique in putting all that pertains to the posterior fossa between two covers. Not an essential purchase for personal libraries, but a good one for training programs.
Clinical Neuroimmunology. Multiple Sclerosis and Related Disorders. 2011. Edited by Syed A. Rizvi, Patricia K. Coyle. Published by Humana Press. 404 pages. C\$240 approx.

\section{Rated irtr}

This textbook is part of the "Current Clinical Neurology" series. To their credit, editors Rizvi and Coyle are well respected experts in the field of MS and Neuroimmunology, and have assembled an excellent group of chapter authors in an attempt to summarize the expansive and ever changing subject of Neuroimmunology. While multiple chapters are dedicated to MS and associated CNS demyelinating disease, there are also chapters on such diverse areas as cancer, epilepsy, dementia, infection and vasculitides to name a few. Particularly well written chapters are those on MRI in MS, NMO and vasculitis. As well, the textbook attempts to provide a primer in the first chapters for those not well versed in immunology fundamentals, albeit in a somewhat dry fashion. Unfortunately, despite a valiant effort, this textbook fails to live up to its goals. In an effort to be all things to all specialties, brief limited chapters on all conceivable neurological diseases are its downfall and the inclusion of some subjects such as CNS infections seems out of place. As is the case for most textbooks, the information becomes obsolete as it is being written, particularly so for this subject matter. I also found this textbook highly redundant (for example, the section on ADEM was already well encompassed in the section on pediatric MS). I was particularly struck by topics that a textbook on MS and neuroimmunology published in 2011 should have, such as more detail on emerging ARMS therapies, disease modifying treatment (DMT) extension trials, and recent breakthroughs in genetics and environmental components of demyelinating disease. On the other hand, a whole chapter dedicated to CCSVI, a presently unproven and controversial theory in MS, is outright odd. I also had considerable concern about the chapter on DMTs with outdated references, no real mention of

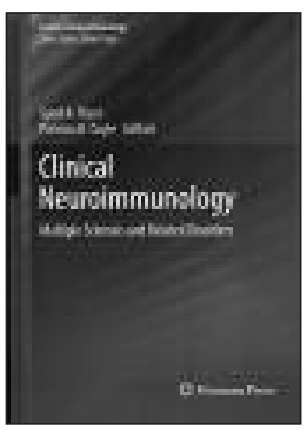
extension studies, comments on combination DMT regiments which are not approved and studies for which have not even yet been published, and a very glancing mention of emerging therapies such as new monoclonal antibodies and stem cell transplantation (the information for which seemed out of date). Unfortunately, an ever-evolving topic such as neuroimmunology coupled with an effort to cover everything thus not covering many topics in sufficient detail, make this textbook relatively uninformative and not sufficient for someone wanting current information on these subjects. Such topics are best studied by reviewing published literature in manuscript databases. I cannot recommend this textbook. 